\title{
Application of simplified PC-SAFT to glycol ethers
}

\section{Avlund, Ane Søgaard; Kontogeorgis, Georgios; Michelsen, Michael Locht}

Published in:

Industrial \& Engineering Chemistry Research

Link to article, DOI:

$10.1021 / \mathrm{ie} 2011406$

Publication date:

2012

Document Version

Publisher's PDF, also known as Version of record

Link back to DTU Orbit

Citation (APA):

Avlund, A. S., Kontogeorgis, G., \& Michelsen, M. L. (2012). Application of simplified PC-SAFT to glycol ethers. Industrial \& Engineering Chemistry Research, 51(1), 547-555. https://doi.org/10.1021/ie2011406

\section{General rights}

Copyright and moral rights for the publications made accessible in the public portal are retained by the authors and/or other copyright owners and it is a condition of accessing publications that users recognise and abide by the legal requirements associated with these rights.

- Users may download and print one copy of any publication from the public portal for the purpose of private study or research.

- You may not further distribute the material or use it for any profit-making activity or commercial gain

- You may freely distribute the URL identifying the publication in the public portal

If you believe that this document breaches copyright please contact us providing details, and we will remove access to the work immediately and investigate your claim. 


\title{
Application of Simplified PC-SAFT to Glycol Ethers
}

\author{
Ane S. Avlund, Georgios M. Kontogeorgis, ${ }^{*}$ and Michael L. Michelsen \\ Center for Energy Resources Engineering (CERE), Department of Chemical and Biochemical Engineering, Technical University of \\ Denmark, Kgs. Lyngby, Denmark
}

ABSTRACT: The simplified PC-SAFT (sPC-SAFT) equation of state is applied for binary glycol ether-containing mixtures, and it is investigated how the results are influenced by inclusion of intramolecular association in the association theory. Three different glycol ethers are examined: 2-methoxyethanol, 2-ethoxyethanol, and 2-butoxyethanol. Vapor-liquid and liquid-liquid equilibria of miscible and immiscible, self- and cross-associating mixtures are considered, including the closed-loop liquid-liquid equilibrium of 2-butoxyethanol-water. The results are finally compared to other association models.

\section{INTRODUCTION}

Glycol ethers are nonionic surfactants with a variety of industrial applications, including a number of applications in the petroleum industry. Glycol ethers contain one hydroxyl group and one or more ether oxygens, and they are the simplest real compounds that can form intramolecular hydrogen bonds. Glycol ethers are therefore interesting candidates for testing the new theory for intramolecular association for SAFT type equations of state ${ }^{1-3}(\mathrm{EoS})$ presented in the $\mathrm{Ph} . \mathrm{D}$. thesis by Avlund ${ }^{4}$ and in a recently published paper. ${ }^{5}$

A lattice theory, which accounts for both inter- and intramolecular association for polyethoxyalcohols, was presented in 1999 by Missopolinou and Panayiotou, ${ }^{6}$ and the theory has been applied for glycol ethers in a number of papers. ${ }^{7-9}$

Because a general theory for intramolecular association in the framework of SAFT was only recently developed, all work with SAFT type EoS so far has been done without considering intramolecular association (explicitly). Garrido et al. ${ }^{10}$ have applied CPA for different binary mixtures of glycol ethers, both self- and cross-associating mixtures, and Garcia-Lisbona et al. ${ }^{11}$ have applied SAFT-HS for 2-butoxyethanol-water.

We have in this work applied sPC-SAFT ${ }^{12}$ for binary glycol ether-containing mixtures. Calculations have been done both with and without inclusion of the new theory for intramolecular association. We have considered miscible and immiscible, selfand cross-associating mixtures.

\section{INTRAMOLECULAR ASSOCIATION}

The association theory used in SAFT only accounts for intermolecular association, but for some systems it is important also to consider the intramolecular interactions. While intermolecular association is interaction between different molecules that are bonded to form dimers or larger chain- or tree-like clusters, intramolecular association is interaction between sites on the same molecule, which leads to the formation of ring structures. The difference between inter- and intramolecular association is illustrated in Figure 1.

Spectroscopy data presented by Brinkley and Gupta ${ }^{13}$ and Missopolinou et al. ${ }^{7}$ have, for dilute mixtures of glycol ethers in $n$-hexane, shown that intramolecular association is dominating as compared to intermolecular association at low glycol ether concentrations. Figure 2 shows the amount of inter- and

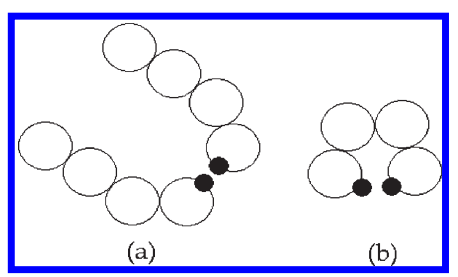

Figure 1. (a) Intermolecular and (b) intramolecular association of model chain molecules.

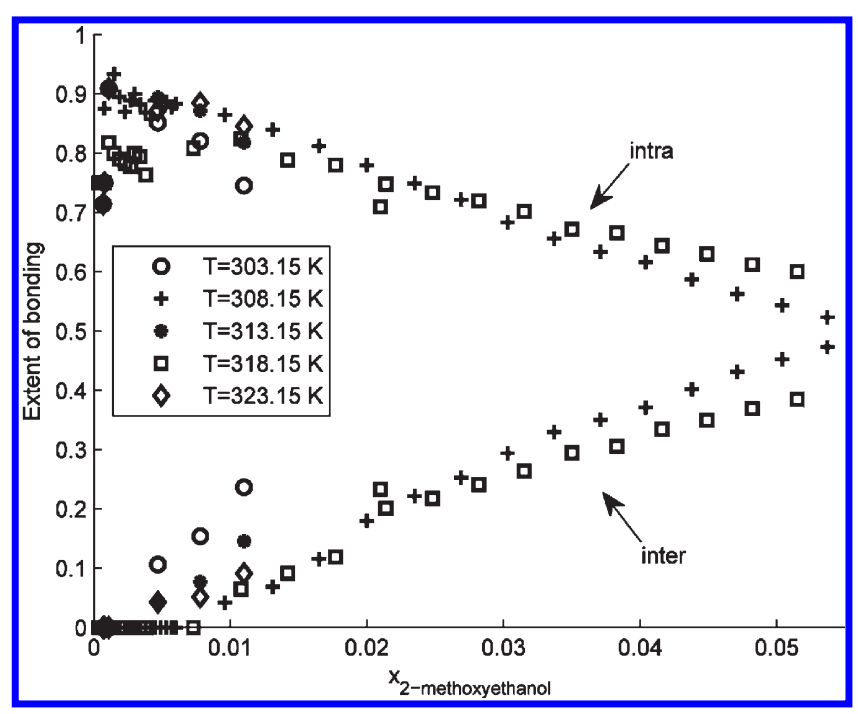

Figure 2. Amount of hydrogen bonds for 2-methoxyethanol-n-hexane, from FT-IR spectroscopy. Experimental data: Brinkley and Gupta ${ }^{13}$ $(T=308.15$ and $318.15 \mathrm{~K})$ and Missopolinou et al. ${ }^{7}(T=303.15$, 313.15 , and $323.15 \mathrm{~K})$.

intramolecular hydrogen bonds for 2-methoxyethanol-n-hexane, measured with FT-IR spectroscopy.

Received: $\quad$ May 27, 2011

Accepted: November 17, 2011

Revised: October 31, 2011

Published: November 17, 2011 
The spectroscopy data show that as the concentration of the glycol ether decreases, the amount of intermolecular bonds also decreases, because the distance between the glycol ether molecules increases, and the probability of being within bonding range of another glycol ether molecule therefore decreases. The intramolecular association does not explicitly depend on concentration (or density), but when the competition from intermolecular association decreases, the amount of intramolecular association increases. It is clear from Figure 2 that there is a large amount of intramolecular bonds in the dilute solution of 2-methoxyethanol in the nonassociating solvent.

A general theory for intramolecular association in the framework of SAFT was presented in a recently published paper by Avlund et al. ${ }^{5}$ and in the Ph.D. thesis by Avlund. ${ }^{4}$ This theory was developed on the basis of previous work by Sear and Jackson ${ }^{14}$ and Ghonasgi and Chapman, ${ }^{15}$ who have developed intramolecular association theories for two associating sites.

According to the theory, the contribution to the Helmholtz free energy from association (inter- as well as intramolecular) is for a pure component given by:

$$
\tilde{a}^{\text {assoc }}=\frac{A^{\text {assoc }}}{N k T}=\ln X_{0}+\frac{1}{2} \sum_{\mathrm{A}}\left(1-X_{\mathrm{A}}\right)-\frac{1}{2} \sum_{\mathrm{A}} \sum_{\mathrm{B} \neq \mathrm{A}} X_{\mathrm{AB}} \Delta_{\mathrm{AB}}^{\mathrm{intra}}
$$

where $X_{\mathrm{A}}$ is the fraction of molecules not bonded at site $\mathrm{A}$, given by:

$$
1=X_{\mathrm{A}}\left[1+\rho \sum_{\mathrm{B}} X_{\mathrm{B}} \Delta_{\mathrm{AB}}^{\mathrm{inter}}\right]+\sum_{\mathrm{B} \neq \mathrm{A}} X_{\mathrm{AB}} \Delta_{\mathrm{AB}}^{\mathrm{intra}}
$$

$X_{0}$ is the monomer fraction (molecules with no bonded sites), which for the specific case, where only two sites (A and B) on a molecule can bond intramolecularly, is given by:

$$
X_{0}=X_{\mathrm{A}}\left(1+\rho \sum_{\mathrm{B}} X_{\mathrm{B}} \Delta_{\mathrm{AB}}^{\mathrm{intra}}\right)
$$

$X_{\mathrm{AB}}$ is the fraction of molecules where both sites $\mathrm{A}$ and $\mathrm{B}$ are unbonded, which for the same specific case is given by:

$$
X_{\mathrm{AB}}=\frac{X_{\mathrm{A}}}{1+\rho \sum_{\mathrm{C}} X_{\mathrm{C}} \Delta_{\mathrm{BC}}^{\mathrm{inter}}}
$$

$\Delta_{\mathrm{AB}}^{\mathrm{inter}}$ and $\Delta_{\mathrm{AB}}^{\mathrm{intra}}$ are the inter- and intramolecular association strengths, respectively. For sPC-SAFT, $\Delta_{\mathrm{AB}}^{\text {inter }}$ is defined as:

$$
\Delta_{\mathrm{AB}}^{\text {inter }}=\frac{\pi}{6} \sigma^{3} g^{\mathrm{HS}}(d) \kappa^{\mathrm{AB}}\left[\exp \left(\frac{\varepsilon^{\mathrm{AB}}}{k T}\right)-1\right]
$$

where $\sigma$ is the segment diameter, $g^{\mathrm{HS}}$ is the partial radial distribution function of hard spheres, and $\kappa^{\mathrm{AB}}$ and $\varepsilon^{\mathrm{AB}}$ are the association volume and energy, respectively. The expression for $\Delta_{\mathrm{AB}}^{\text {intra }}$ used in this work was taken from Sear and Jackson. ${ }^{14}$ They suggest setting the intramolecular association strength equal to the intermolecular association strength multiplied by $W_{n}$ :

$$
\Delta_{\mathrm{AB}}^{\mathrm{intra}}=W_{n} \Delta_{\mathrm{AB}}^{\mathrm{intra}}=W_{n} \frac{\pi}{6} \sigma^{3} g^{\mathrm{HS}}(d) \kappa^{\mathrm{AB}}\left[\exp \left(\frac{\varepsilon^{\mathrm{AB}}}{k T}\right)-1\right]
$$

where $W_{n}$ is the value of the end-to-end distribution function of a freely jointed chain of $n$ links, when the end links are the length of

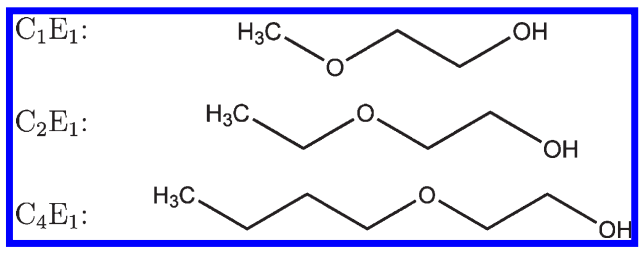

Figure 3. Structures of 2-methoxyethanol $\left(\mathrm{C}_{1} \mathrm{E}_{1}\right)$, 2-ethoxyethanol $\left(\mathrm{C}_{2} \mathrm{E}_{1}\right)$, and 2-butoxyethanol $\left(\mathrm{C}_{4} \mathrm{E}_{1}\right)$.

one link apart. $W_{n}$ is defined as:

$$
W_{n}=\frac{n(n-1)}{\sigma^{3} 8 \pi} \sum_{j=0}^{l} \frac{(-1)^{j}}{j !(n-j) !}\left[\frac{n-1-2 j}{2}\right]^{n-2}
$$

where $l$ is the smallest integer satisfying:

$$
l \geq \frac{n-1}{2}-1
$$

(There was a misprint in the expression for $W_{n}$ in the paper by Sear and Jackson, which has been corrected in eq 7.) The expression for the intramolecular association strength in eq 6 implies using identical association parameters for inter- and intramolecular association, which is an approximation, but also maintains the number of adjustable parameters. In this way, the new theory for intramolecular association can be compared to "normal" sPC-SAFT (without intramolecular association) with the same number of parameters, and thereby on equal terms.

\section{RESULTS WITH SPC-SAFT}

Association Scheme. There is consensus in the SAFT community of using the nomenclature for association schemes presented by Huang and Radosz, ${ }^{16}$ but that nomenclature is not systematic. Instead, we use a nomenclature, where association schemes are defined by the total number of associating sites, $x$, the number of proton acceptor sites, $y$, and the number of proton donor sites, $z$, written as $x(y: z)$. This definition is identical to the one used by Yarrison and Chapman ${ }^{17}$ and by Avlund et al. ${ }^{18}$

Three glycolethers have been considered in this work: 2-methoxyethanol $\left(\mathrm{C}_{1} \mathrm{E}_{1}\right)$, 2-ethoxyethanol $\left(\mathrm{C}_{2} \mathrm{E}_{1}\right)$, and 2-butoxyethanol $\left(\mathrm{C}_{4} \mathrm{E}_{1}\right)$. The structures of the three glycol ethers are shown in Figure 3. The multifunctionality of glycol ethers gives the option of several association schemes, and different association schemes have been used for modeling glycol ethers with SAFT type theories in the literature; Garrido et al. ${ }^{10}$ applied both the $2(1: 1)$ and the $3(2: 1)$ schemes, while Garcia-Lisbona et al. ${ }^{11}$ used a complex scheme with three sites for each ether oxygen and three sites for the hydroxyl group, where some sites are only allowed to cross-associate, while others can self- as well as cross-associate. (The association scheme used by Garcia-Lisbona et al. is actually an implicit way of accounting for intramolecular association.) The 3 (2:1) scheme (one proton donor site and one proton acceptor site for the hydroxyl group and one proton acceptor site for the ether oxygen) is the simplest reasonable scheme for which intramolecular association is possible, and only this scheme is used in this work. A similar definition of the associating sites is used in the work with lattice theories for polyethoxyalcohols. ${ }^{6-9}$

Parameter Estimation. Two parameter sets have been estimated for each of the three glycol ethers: one set for regular SPC$\mathrm{SAFT}^{12}$ and one for SPC-SAFT plus intramolecular association. 
Table 1. PC-SAFT Parameters for Three Glycol Ethers, Temperature Interval Used in the Parameter Estimation, and Average Relative Deviation (ARD\%) between SPC-SAFT and DIPPR Correlations ${ }^{19}$ in the Temperature Range of the Estimation

\begin{tabular}{|c|c|c|c|c|c|c|c|c|}
\hline & \multirow[b]{2}{*}{$\sigma[\AA]$} & \multirow[b]{2}{*}{$\varepsilon[\mathrm{K}]$} & \multirow[b]{2}{*}{$m$} & \multirow[b]{2}{*}{$\varepsilon^{\mathrm{AB}}[\mathrm{K}]$} & \multirow[b]{2}{*}{$\kappa^{\mathrm{AB}}$} & \multirow[b]{2}{*}{$T[\mathrm{~K}]$} & \multicolumn{2}{|c|}{ ARD $\%^{a}$} \\
\hline & & & & & & & $P^{\mathrm{sat}}$ & $V_{\mathrm{m}}^{\mathrm{L}}$ \\
\hline $\mathrm{C}_{1} \mathrm{E}_{1}$ inter & 2.958 & 187.69 & 3.956 & 1698.3 & 0.4975 & $282-508$ & 2.31 & 0.21 \\
\hline $\mathrm{C}_{1} \mathrm{E}_{1}$ intra & 2.779 & 187.97 & 4.768 & 1546.9 & 0.5603 & $310-508$ & 1.71 & 0.30 \\
\hline $\mathrm{C}_{2} \mathrm{E}_{1}$ inter & 2.999 & 189.56 & 4.693 & 1289.2 & 0.9179 & $285-512$ & 2.28 & 0.24 \\
\hline $\mathrm{C}_{2} \mathrm{E}_{1}$ intra & 2.921 & 187.95 & 5.055 & 1465.7 & 0.6375 & $313-512$ & 1.74 & 0.28 \\
\hline $\mathrm{C}_{4} \mathrm{E}_{1}$ inter & 3.518 & 257.63 & 4.194 & 1206.7 & 0.0446 & $317-571$ & 0.39 & 0.23 \\
\hline $\mathrm{C}_{4} \mathrm{E}_{1}$ intra & 3.252 & 203.41 & 5.127 & 1465.7 & 0.6375 & $317-571$ & 4.18 & 0.18 \\
\hline
\end{tabular}

sPC-SAFT plus intramolecular association means that the new association term presented by Avlund et al. ${ }^{5}$ (eq 1), where intramolecular association is included, is used instead of the usual association term from sPC-SAFT, while the remaining terms are the same for the two models. Using the expression for the intramolecular association strength in eq 6 from Sear and Jackson $^{14}$ enables us to use identical values of the association parameters for inter- and intramolecular association $\left(\varepsilon^{\text {inter }}=\varepsilon^{\text {intra }}\right.$ and $\left.\kappa^{\text {inter }}=\kappa^{\text {intra }}\right)$. The two models therefore have the same five adjustable pure component parameters. The $W_{n}$ parameter in eq 7 depends on the number of links (bonds), $n$, between the intramolecularly associating sites and on the segment diameter. The number of links between the ether oxygen and the hydroxyl hydrogen in the three glycol ethers considered here is 4 , giving the following value of $W_{n}$ :

$$
W_{4}=0.0248 / \sigma^{3}
$$

The pure component sPC-SAFT parameters were obtained by fitting DIPPR ${ }^{19}$ correlations of pure component vapor pressure and liquid density. The parameters for the three glycol ethers are listed in Table 1. Inter indicates parameter sets for sPC-SAFT without intramolecular association (sPC-SAFT-inter), and intra indicates sets for sPC-SAFT plus intramolecular association (sPC-SAFT-intra).

When parameters were estimated for $\mathrm{C}_{4} \mathrm{E}_{1}$ by fitting pure component vapor pressure and liquid density in the temperature interval corresponding to $T_{\mathrm{r}}=0.5-0.9$, relatively small association parameters were obtained as compared to the other two glycol ethers, as seen for the inter parameter set in Table 1. The only difference between the two models used in this work is related to association, and the two models are therefore very similar if there is little association. Almost identical inter and intra parameters were therefore obtained, and the two parameter sets resulted in very similar results. A further discussion is presented in Chapter 6 of the Ph.D. thesis by Avlund. ${ }^{4}$

On the basis of the structures of the three glycol ethers, there is no reason why the association of $\mathrm{C}_{4} \mathrm{E}_{1}$ should be significantly weaker than for the other two glycol ethers, and a new intra parameter set was therefore estimated on the basis of the association parameters of $\mathrm{C}_{2} \mathrm{E}_{1}$. This procedure was decided on the basis of an observation of very similar association strengths for $C_{1} E_{1}$ and $\mathrm{C}_{2} \mathrm{E}_{1}$ for sPC-SAFT-intra. It should be noted that the error in vapor pressure with the intra parameters for $\mathrm{C}_{4} \mathrm{E}_{1}$ is around $4 \%$, which is significantly higher than that for the other parameter

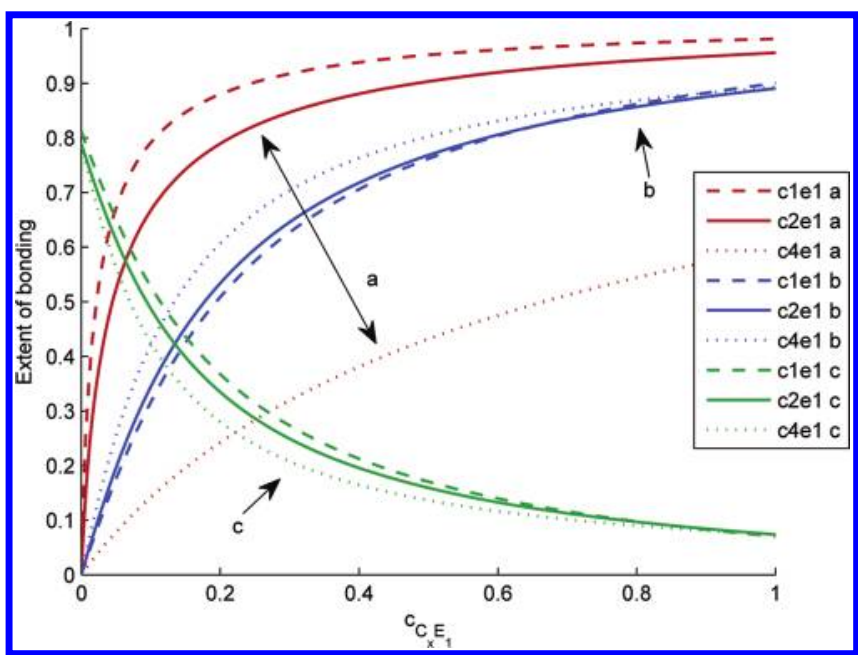

Figure 4. Amount of inter- and intramolecular bonds in $\mathrm{C}_{x} \mathrm{E}_{1}-$ $n$-hexane. $a=$ intermolecular association with inter parameters, $b=$ intermolecular association with intra parameters, and $c=$ intramolecular association with intra parameters.

sets, and which of course is dissatisfactory. The vapor pressure error with the inter parameters for $\mathrm{C}_{4} \mathrm{E}_{1}$ is surprisingly small as compared to the other sets, and the $m$ parameter in that set is smaller than $m$ in the inter set for $\mathrm{C}_{2} \mathrm{E}_{1}$, which of course does not make sense physically. (Because of the intercorrelation of the parameters, $\sigma$ for the $\mathrm{C}_{4} \mathrm{E}_{1}$ inter set is accordingly considerably larger than for the other sets.) It would therefore be advantageous to reconsider the parameter estimation for $\mathrm{C}_{4} \mathrm{E}_{1}$, maybe in combination with the parameters for $\mathrm{C}_{1} \mathrm{E}_{1}$ and $\mathrm{C}_{2} \mathrm{E}_{1}$, but that is outside the scope of this work, and the presented parameters have been used for an initial investigation of $\mathrm{C}_{4} \mathrm{E}_{1}$.

The six parameter sets are compared by considering the amount of inter- and intramolecular bonds predicted in a mixture of $\mathrm{C}_{x} \mathrm{E}_{1}$ and $n$-hexane. The results are shown in Figure 4. The calculations were done for a single liquid phase at $P=1.013 \mathrm{bar}$ and $T=300 \mathrm{~K}$, with $k_{i j}=0$.

As expected from the values of the association parameters, the inter parameters for $\mathrm{C}_{4} \mathrm{E}_{1}$ predict significantly less intermolecular association than do the other parameter sets. The amount of bonds predicted with the inter parameters for $\mathrm{C}_{1} \mathrm{E}_{1}$ and $\mathrm{C}_{2} \mathrm{E}_{1}$ differs somewhat, while the intra parameter sets for the three glycol ethers give rather similar results. For $\mathrm{C}_{1} \mathrm{E}_{1}$ and $\mathrm{C}_{2} \mathrm{E}_{1}$, sPCSAFT-intra predicts less intermolecular association than does sPC-SAFT-inter, because of the competition from intramolecular association.

There are large uncertainties involved in the estimation of the amount of bonds with spectroscopy, and we have therefore decided not to make a quantitative comparison between sPCSAFT and experimental data, but a qualitative comparison of Figures 2 and 4 shows that the results with sPC-SAFT-intra predict the same trends as the spectroscopic data show.

VLE and LLE Results for Self-Associating Mixtures. We first consider binary mixtures of glycol ethers and an inert (nonassociating) compound. The PC-SAFT parameters for the alkanes were taken from Gross and Sadowski. ${ }^{20}$ Figures 5 and 6 show VLE and LLE results for $\mathrm{C}_{1} \mathrm{E}_{1}-n$-hexane. sPC-SAFT-inter does not predict $\left(k_{i j}=0\right)$ LLE for this system, whereas sPCSAFT-intra predicts a too high upper critical solution temperature (UCST). It is not possible with either model to correlate (fitting a 


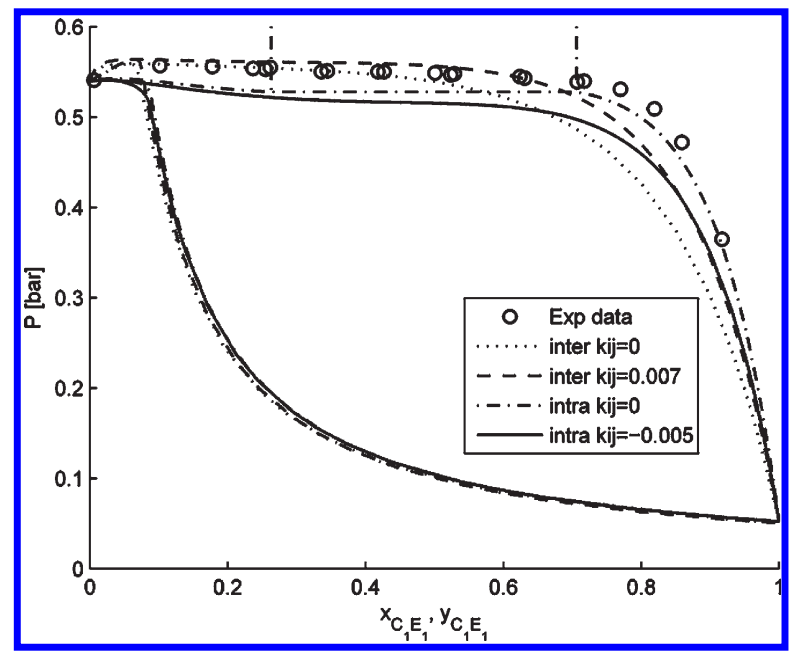

Figure 5. $\mathrm{C}_{1} \mathrm{E}_{1}-n$-hexane VLE at $T=323.15 \mathrm{~K}$. Experimental data: Dolch et al. ${ }^{21}$

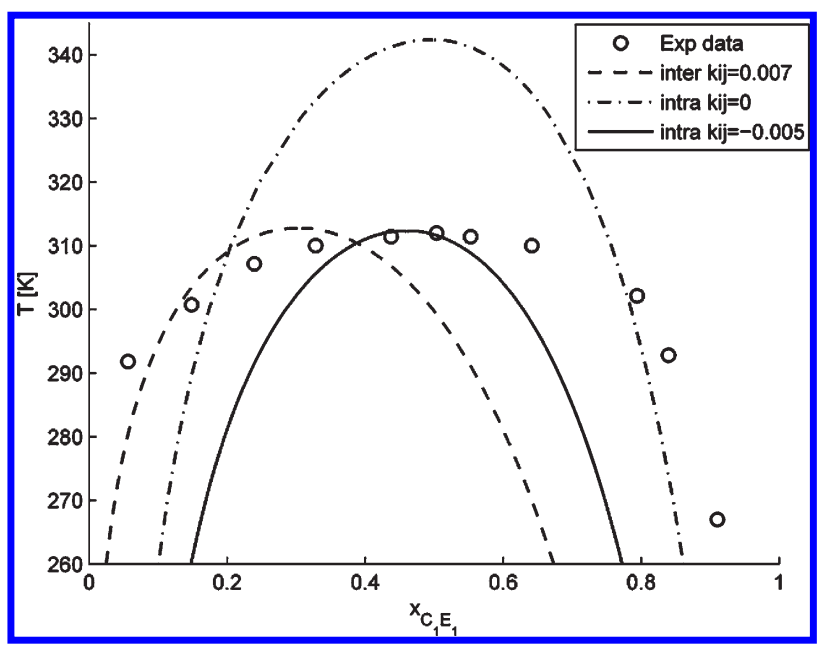

Figure 6. $\mathrm{C}_{1} \mathrm{E}_{1}-n$-hexane LLE at $P=1.013$ bar. Experimental data: Bijl et al. ${ }^{22}$

binary interaction parameter, $k_{i j}$ ) the very flat upper part of the immiscibility area. If the interaction parameter is fitted to match the UCST, the estimated immiscibility area becomes too small (narrow) with both models. The results with sPC-SAFT-intra deviate similarly for the two phases, while the results with sPCSAFT-inter are shifted to lower glycol ether concentrations. There is only a small single phase temperature interval between the VLE and LLE data, and because SPC-SAFT-intra overestimates UCST it predicts VLLE at $T=323.15 \mathrm{~K}$.

The same $k_{i j}$ values are used for VLE and LLE for $\mathrm{C}_{1} \mathrm{E}_{1}-$ $n$-hexane, but it is not possible to match all of the VLE data points with either model even with a $k_{i j}$ optimized for VLE. sPC-SAFTinter can only match the data points at medium and low glycol ether concentrations, while SPC-SAFT-intra matches the glycol ether rich part well, but is disturbed by the model prediction of LLE at lower concentrations. Similar results are obtained for $\mathrm{C}_{1} \mathrm{E}_{1}-n$-heptane.

VLE results are shown for $\mathrm{C}_{2} \mathrm{E}_{1}-n$-octane in Figure 7. sPCSAFT-inter and sPC-SAFT-intra both predict the azeotrope of this mixture, although both models slightly underpredict the azeotrope temperature. The error with sPC-SAFT-inter is twice

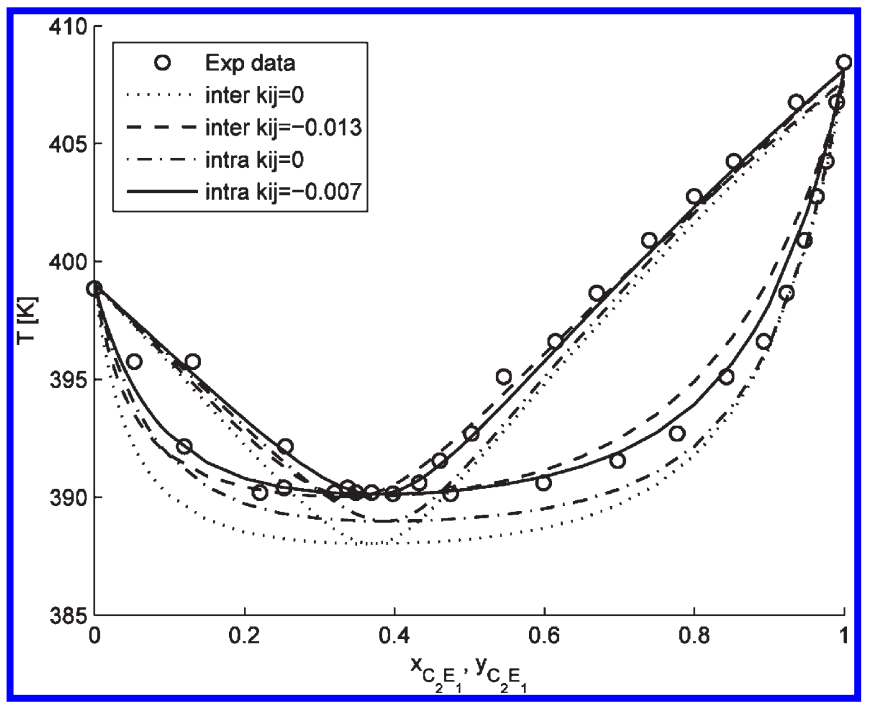

Figure 7. $\mathrm{C}_{2} \mathrm{E}_{1}-n$-octane VLE at $P=1.013$ bar. Experimental data: Murti and Van Winkle. ${ }^{23}$

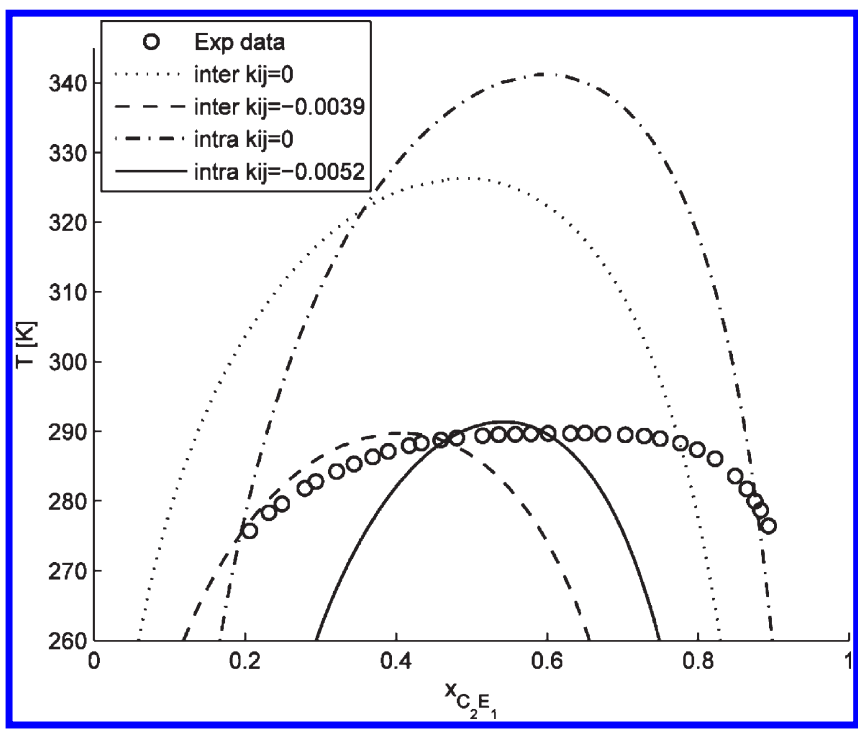

Figure 8. $\mathrm{C}_{2} \mathrm{E}_{1}-n$-dodecane LLE at $P=1.013$ bar. Experimental data: Rubio et al. ${ }^{24}$

the error with sPC-SAFT-intra. It is possible with both models to correlate the data including the azeotrope satisfactorily by fitting a $k_{i j}$, although slightly better results are obtained with sPC-SAFTintra than with sPC-SAFT-inter.

Figure 8 shows the LLE results for $\mathrm{C}_{2} \mathrm{E}_{1}-n$-dodecane. Both models predict LLE for this system, and both overestimate the UCST. The results with an interaction parameter fitted to match UCST are similar to the results for $\mathrm{C}_{1} \mathrm{E}_{1}-n$-hexane. The estimated immiscibility areas are too narrow, and the sPCSAFT-inter results are shifted toward lower glycol ether concentrations as compared to the sPC-SAFT-intra results, which lie in the center of the experimental immiscibility area.

The results for $\mathrm{C}_{4} \mathrm{E}_{1}-n$-octane are shown in Figure 9. Here, sPC-SAFT-intra gives significantly better results for $k_{i j}=0$, and a better match to the liquid curve with an optimal $k_{i j}$ than sPCSAFT-inter. sPC-SAFT-inter correlates the vapor curve slightly 


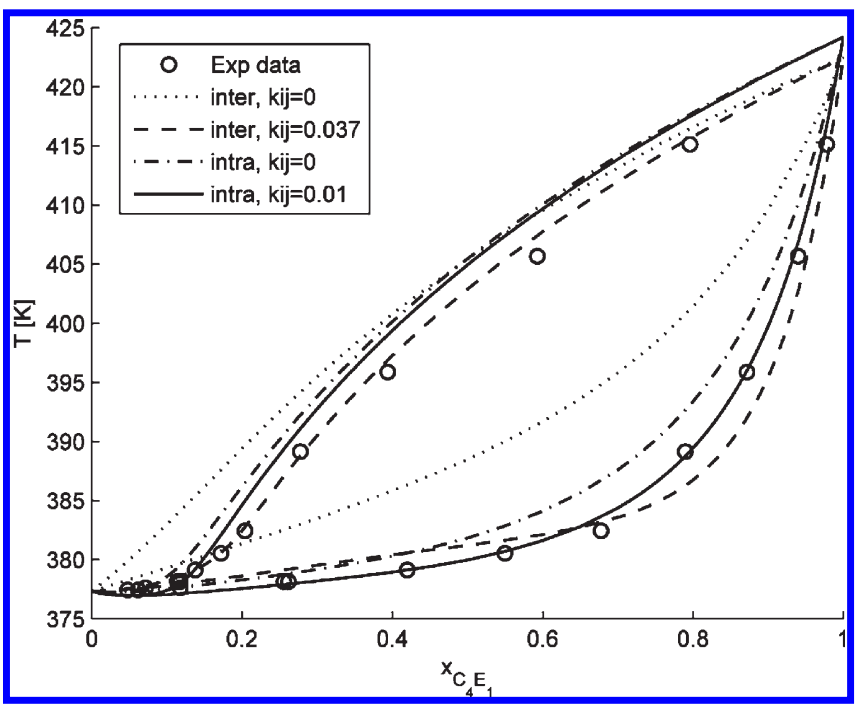

Figure 9. $\mathrm{C}_{4} \mathrm{E}_{1}-n$-octane VLE at $P=0.533$ bar. Experimental data: Komatsu et al. ${ }^{25}$

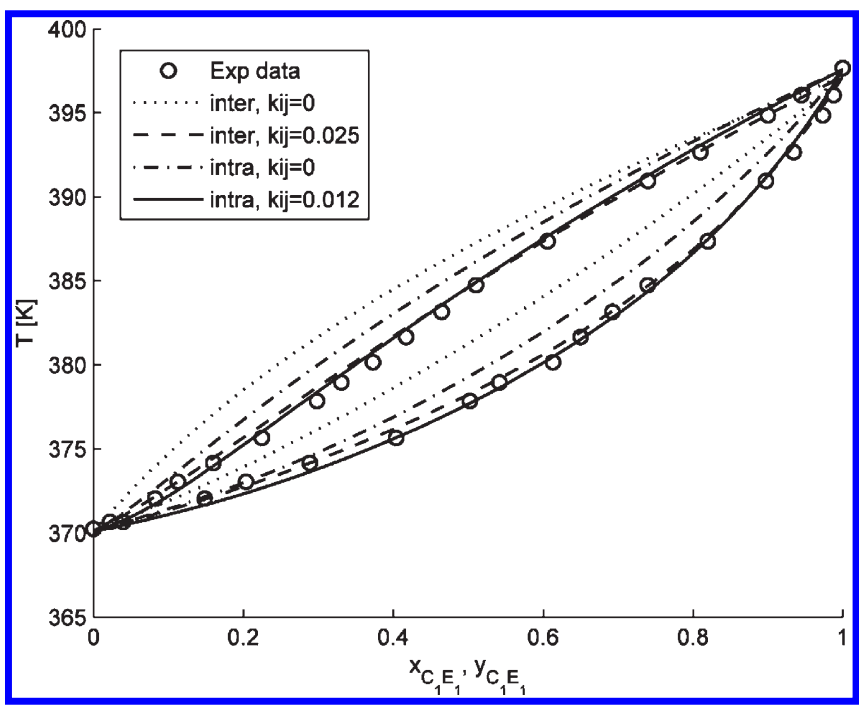

Figure 10. $\mathrm{C}_{1} \mathrm{E}_{1}$ - propanol VLE at $P=1.013$ bar. Experimental data: Chandak et al. ${ }^{28}$

better than SPC-SAFT-intra, which is a result of the large error in vapor pressure for pure $\mathrm{C}_{4} \mathrm{E}_{1}$ with sPC-SAFT-intra.

VLE and LLE Results for Cross-Associating Mixtures. Next, we consider binary mixtures of glycol ethers with other associating compounds. The Elliott combining rule ${ }^{26}$ was used in the calculations.

Figure 10 shows the results for $\mathrm{C}_{1} \mathrm{E}_{1}$ - propanol with $k_{i j}=0$ as well as with fitted $k_{i j}$. Propanol was modeled using the 2 (1:1) scheme and generalized alkanol parameters from Grenner et al. ${ }^{27}$ sPC-SAFT-intra gives a better prediction for this mixture than does SPC-SAFT-inter, but the cross-attractions between the two compounds are overestimated by both models. Both models correlate the system very satisfactorily with a small positive $k_{i j}$. sPC-SAFT-inter needs a larger $k_{i j}$ than sPC-SAFT-intra because of the larger error in the predictions.

The results for $\mathrm{C}_{2} \mathrm{E}_{1}$-water are shown in Figures 11 and 12 . Water was modeled with the 4 (2:2) scheme and the parameters from Grenner et al. ${ }^{29}$ The figures show the results with both

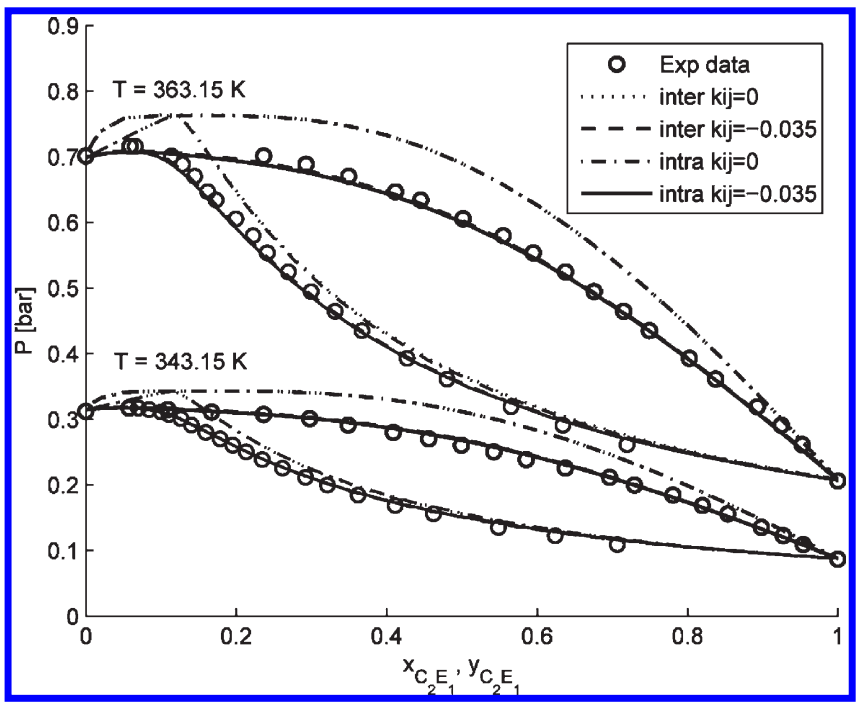

Figure 11. $C_{2} E_{1}-$ water VLE at $T=343.15$ and $363.15 \mathrm{~K}$. Experimental data: Chiavone-Filho et al. ${ }^{30}$

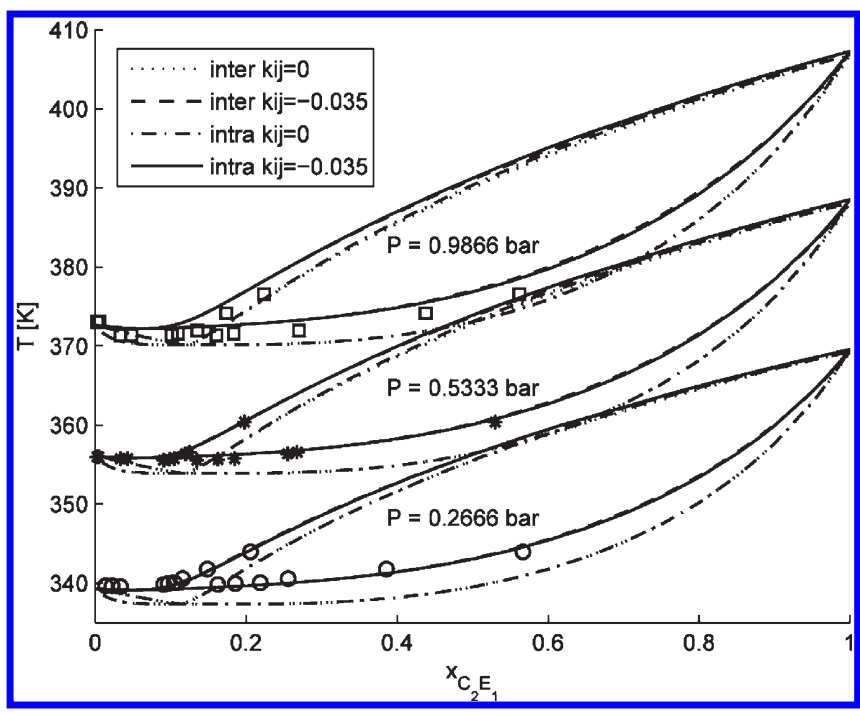

Figure 12. $\mathrm{C}_{2} \mathrm{E}_{1}-$ water VLE at $P=0.2666,0.5333$, and 0.9866 bar. Experimental data: Boublik and Kuchynka. ${ }^{31}$

sPC-SAFT-inter and sPC-SAFT-intra, but the results with the two models are indistinguishable. As seen from the figures, sPCSAFT very satisfactorily models this system, and a single $k_{i j}$ is sufficient to match the experimental data, including the azeotrope, at all temperatures and pressures investigated here.

The last cross-associating mixture with a glycol ether to be considered here is $\mathrm{C}_{4} \mathrm{E}_{1}$-water. This is a very interesting mixture, both because of its industrial applications and because it exhibits closed-loop LLE. The closed-loop LLE is a result of the competition between energetic and entropic effects. At temperatures above UCST, the system will minimize its free energy by maximizing its compositional and orientational entropy. At intermediate temperatures, the entropy is less important, and the system will consequently try to minimize the enthalpy. The system will therefore split into two immiscible liquid phases because of the weak van der Waals forces between unlike molecules. At temperatures below the lower critical solution 


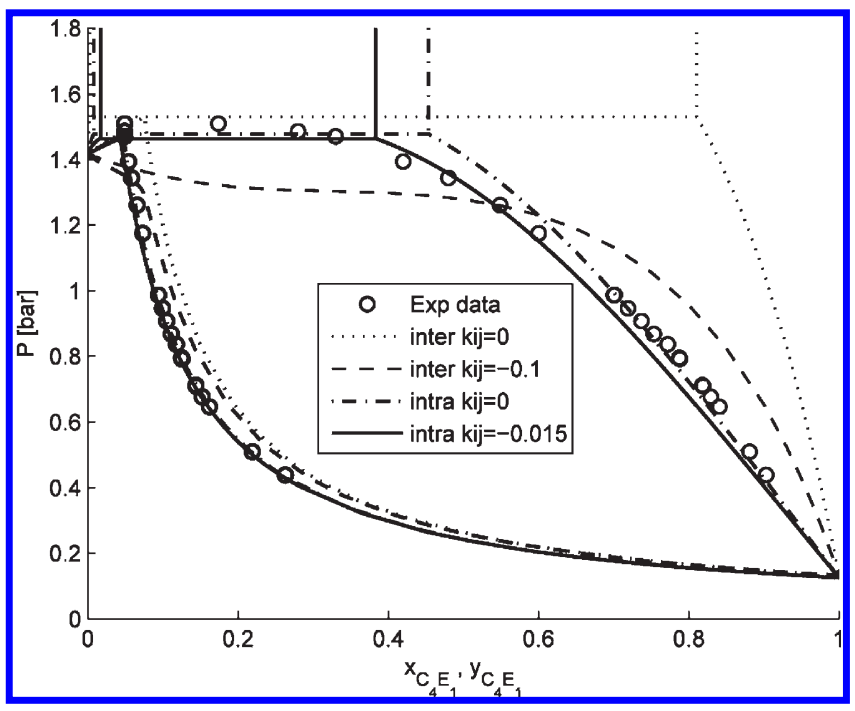

Figure 13. $\mathrm{C}_{4} \mathrm{E}_{1}$-water VLE at $T=383.15 \mathrm{~K}$. Experimental data: Schneider and Wilhelm. ${ }^{32}$

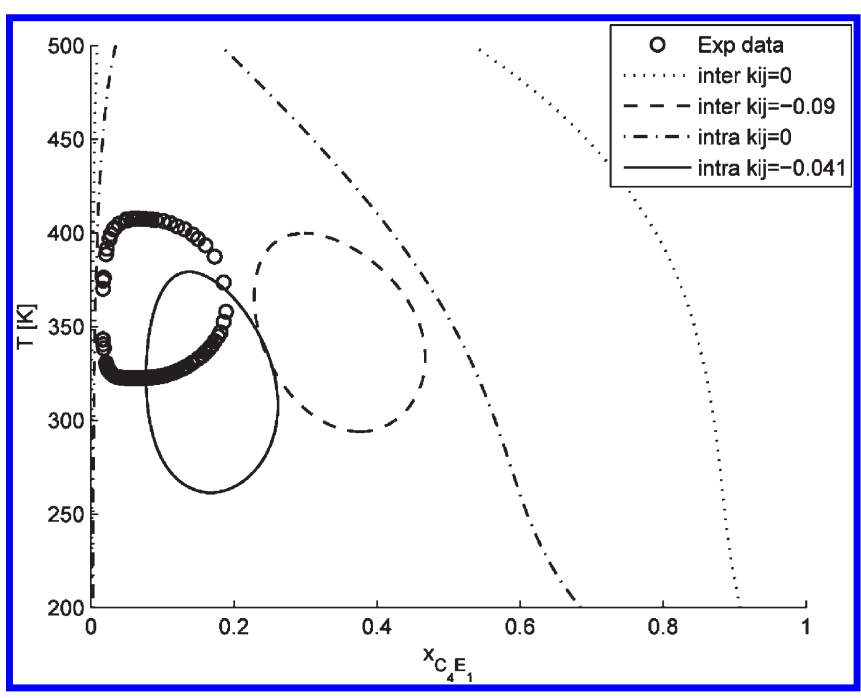

Figure 14. $\mathrm{C}_{4} \mathrm{E}_{1}$-water $\mathrm{LLE}$ at $P=10.13$ bar. Experimental data: Aizpiri et al. ${ }^{33}$

temperature (LCST), cross-association becomes strong enough to overcome the unfavorable enthalpy and orientational entropy and to keep the mixture in one phase. ${ }^{11}$ Because the entropic effects are essential for the formation of closed-loop LLE, the highly anisotropic hydrogen bonds must be treated in the theory to describe the phase behavior. An EoS like sPC-SAFT is therefore in theory capable of modeling closed-loop LLE.

Figure 13 shows the VLE results and Figure 14 the LLE results with sPC-SAFT for this mixture. sPC-SAFT-intra gives significantly better VLE results for this mixture than does SPC-SAFTinter, but the predicted VLLE area is in both cases larger than what the experimental data suggest, which disturbs the results for the liquid curve. It is not possible to fit a $k_{i j}$ to match the entire liquid curve.

Neither of the models are capable of correlating the data points for the closed-loop LLE of this mixture. Both models significantly overestimate the immiscibility area both in temperature and composition, and fitting one interaction parameter is not enough

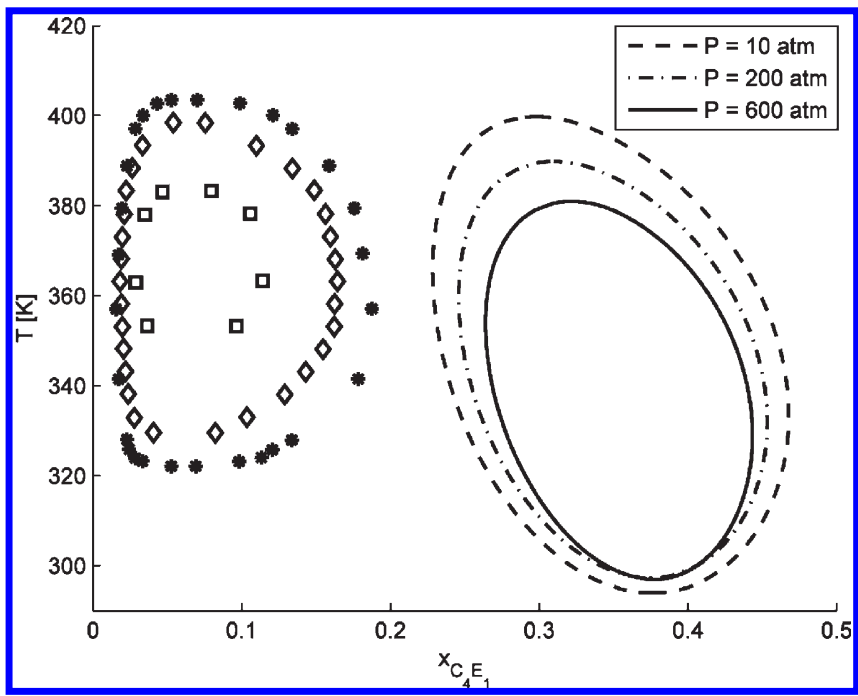

Figure 15. Results with sPC-SAFT-inter for $\mathrm{C}_{4} \mathrm{E}_{1}$-water LLE at $P=10,200$, and $600 \mathrm{~atm}$. Experimental data: Schneider, ${ }^{34}$ taken from Knudsen et al.; ${ }^{35} 10 \mathrm{~atm}(*), 200 \mathrm{~atm}(\diamond)$, and $600 \mathrm{~atm}(\square)$.

to match the experimental data points. It is possible with sPCSAFT-inter to get a good match of UCST and LCST with the same $k_{i j}$, but then the composition of the water-rich phase is overestimated with an order of magnitude. sPC-SAFT-intra gives a significantly better correlation of the composition than does sPC-SAFT-inter, but UCST and LCST are not as well matched as with sPC-SAFT-inter. (The $k_{i j}$ values for LLE were fitted to give an immiscibility area of approximately the same size as the experimental data shows). The fact that sPC-SAFT-inter predicts a significantly larger immiscibility area than what the experimental data show could be a result of the weak association with the $\mathrm{C}_{4} \mathrm{E}_{1}$ inter parameters, because more cross-association presumably will increase the miscibility between $\mathrm{C}_{4} \mathrm{E}_{1}$ and water. This was part of the reasoning behind the new intra parameter set, and it is clear from Figures 13 and 14 that stronger association has improved the results, even though the immiscibility area predicted with sPC-SAFT-intra is also significantly larger than what the experimental data suggest.

Among the applications of glycol ethers in the petroleum industry is enhanced oil recovery (EOR) by chemical flooding of the oil reservoirs. Because the reservoir pressures are usually significantly higher than atmospheric pressure, it is important to know how the efficiency of the flooding depends on pressure. As a step in that investigation, it is also of importance to know how the miscibility of $\mathrm{C}_{4} \mathrm{E}_{1}$ and water is influenced by pressure. Schneider ${ }^{34}$ has presented extrapolated LLE data for this mixture at different pressures. Figures 15 and 16 show the results with sPC-SAFT-inter and sPC-SAFT-intra, respectively, at $P=10$, 200 , and $600 \mathrm{~atm}$.

sPC-SAFT-inter predicts qualitatively the correct pressure dependency, but the experimental data show a stronger pressure dependency than what is predicted with the model. Moreover, the predicted LLE area shifts toward lower temperatures as the pressure increases, as compared to the experimental data.

sPC-SAFT-intra gives a better prediction of the pressure dependency than does SPC-SAFT-inter. Where sPC-SAFT-inter gives a weaker pressure dependency than the experimental data show, the pressure dependency of sPC-SAFT-intra for $\mathrm{C}_{4} \mathrm{E}_{1}$ is very similar to the experimental pressure dependency. 


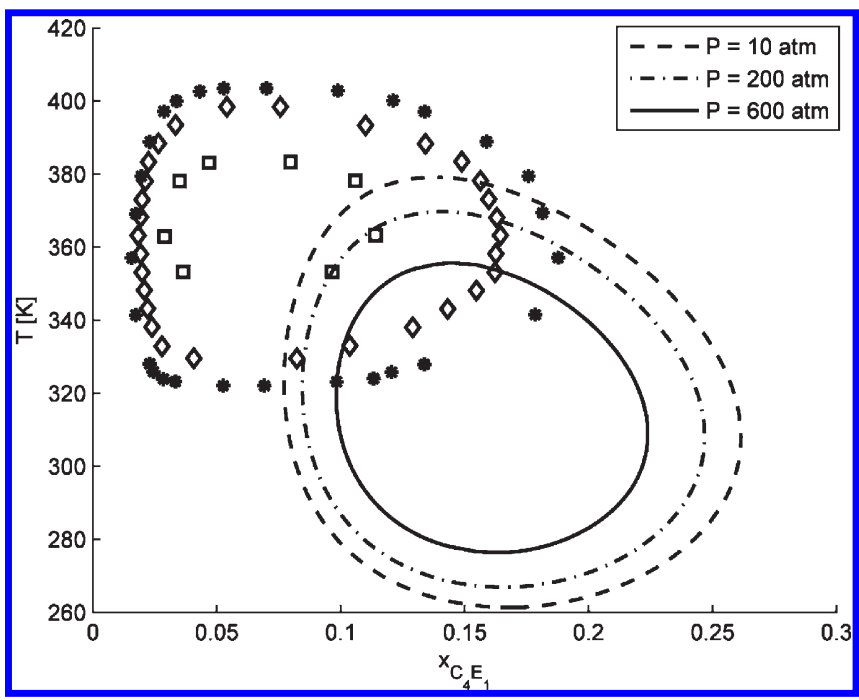

Figure 16. Results with sPC-SAFT-intra for $\mathrm{C}_{4} \mathrm{E}_{1}-$ water LLE at $P=10$, 200 , and $600 \mathrm{~atm}$. Experimental data: Schneider, ${ }^{34}$ taken from Knudsen et al.; ${ }^{35} 10 \mathrm{~atm}(*), 200 \mathrm{~atm}(\diamond)$, and $600 \mathrm{~atm}(\square)$.

It is important to note that the difference in the results with sPC-SAFT-inter and sPC-SAFT-intra for $\mathrm{C}_{4} \mathrm{E}_{1}$ is mainly a result of the different parameter estimation procedures. Estimating a new parameter set for SPC-SAFT-intra with stronger association significantly improved the results (the original parameter set gave results almost identical to the ones presented for sPC-SAFTinter), confirming that the parameters obtained from pure $\mathrm{C}_{4} \mathrm{E}_{1}$ data underestimated the association. On the basis of this observation, it is believed that better parameters can also be obtained for sPC-SAFT-inter, if stronger association is enforced. On the basis of the results with SPC-SAFT-intra presented here, it is believed that if a parameter set was fitted to match the experimental LLE data points at $P=1$ bar, then sPC-SAFT-intra could predict the data points at higher pressures. This has not been investigated in this work.

\section{COMPARISON WITH OTHER ASSOCIATION MODELS}

The results presented for glycol ethers with lattice theories in the literature are mainly for excess enthalpies, and phase equilibrium for mixtures with $\mathrm{CO}_{2}$, and it is therefore not possible to compare the two approaches presented in this work.

Garrido et al., ${ }^{10}$ on the other hand, considered many of the same systems that were investigated here. They interestingly found that 2 sites for glycol ethers in most cases gave better results than 3 sites, and a comparison shows that the $2(1: 1)$ results with CPA are very similar to the results with 3 (2:1) with sPC-SAFT-inter. The only system where that is not the case is $\mathrm{C}_{4} \mathrm{E}_{1}$-water. Here, Garrido et al. found that the 2 (1:1) scheme gives good results for VLE, and the 3 (2:1) scheme gives good results for LLE, and the results are in both cases significantly better than the results obtained with sPC-SAFT, whether intramolecular association is included or not. It was not possible with CPA to obtain good results for both VLE and LLE with the same association scheme for this mixture.

The closed-loop LLE of $\mathrm{C}_{4} \mathrm{E}_{1}$ - water was also investigated by Garcia-Lisbona et al., ${ }^{11}$ who found it necessary to fit the number and type of associating sites, as well as the parameters to the LLE data to match the experimental data points. With parameters and an association scheme obtained in this way, they get LLE results similar to the 2 (1:1) CPA results. Reasonable VLE results were obtained using the $k_{i j}$ fitted for LLE.

\section{CONCLUSION}

sPC-SAFT was in general found to perform satisfactorily for glycol ether-containing mixtures, both with and without the new theory for intramolecular association. The predicted results are in most cases better when intramolecular association is included, especially for LLE, while similar results were obtained with a fitted binary interaction parameter. SPC-SAFT was in general found to give very good results for miscible mixtures and reasonably good results for immiscible mixtures, except for the mixture of $\mathrm{C}_{4} \mathrm{E}_{1}$-water, for which the results were not satisfactory.

On the basis of the very similar association strengths for $\mathrm{C}_{1} \mathrm{E}_{1}$ and $\mathrm{C}_{2} \mathrm{E}_{1}$ with sPC-SAFT-intra, a new parameter set was estimated for $\mathrm{C}_{4} \mathrm{E}_{1}$ with the association parameters from $\mathrm{C}_{2} \mathrm{E}_{1}$. With these parameters, sPC-SAFT-intra was found to give significantly better VLE results for $\mathrm{C}_{4} \mathrm{E}_{1}$-water, as well as better LLE results than $\mathrm{PPC}$-SAFT-inter. This difference between $\mathrm{SPC}$ SAFT-inter and sPC-SAFT-intra for $\mathrm{C}_{4} \mathrm{E}_{1}$ is, however, mainly caused by the difference in the parameter estimation, and not by the difference in the theories. The five adjustable parameters may have a larger influence on the performance of the model than improvements in the theory (at least for this type of calculations), which explains the similar results obtained in this work with sPCSAFT with and without intramolecular association. There is thus no significant gain in using the new, more extensive theory for this kind of calculations, but the new theory is expected to be of more importance for other types of calculations, including derivative properties, multicomponent mixtures, and group contribution methods for multifunctional compounds. If, for example, the parameters for hydroxyl and amine groups are estimated for alcohols and amines, and applied for glycols and alkanolamines, then the effects that are only present for multifunctional compounds, like intramolecular association, must be accounted for.

A comparison between SPC-SAFT-inter and CPA showed that the results with SPC-SAFT (without intramolecular association) with the 3 (2:1) scheme and CPA with the $2(1: 1)$ scheme are similar.

\section{APPENDIX: DERIVATIVES OF THE HELMHOLTZ FREE ENERGY}

This Appendix will present expressions for the derivatives of the Helmholtz free energy needed to calculate pressure and chemical potential for the theory for intramolecular association presented by Avlund et al. ${ }^{5}$ Michelsen and Hendriks ${ }^{36}$ presented a simple way to calculate these derivatives when only intermolecular association is considered, and the same approach is used here. The derivatives are given for the case where only two sites on a molecule can associate intramolecularly.

The nomenclature used in this Appendix is convenient for programming, because it reduces the number of sums, as compared to the normal SAFT nomenclature. All sites in the system are listed after each other, starting with the sites on component 1 , followed by the sites on component 2 , and so on. Sites are referred to by the number in the list. $X A_{i}$ is thus the fraction of sites $i$ not bonded, and $X A B_{i j}$ is the fraction of molecules where both sites $i$ and $j$ are not bonded. $x_{k}$ is the component mole fraction, while $x_{i}$ is the mole fraction of site $i$, 
which is equal to the mole fraction of the component, which site $i$ belongs to, multiplied by the repetition factor of the site. Sums over $i$ and $j$ are implicitly over all sites in the system if not otherwise stated, except for intramolecular interactions, where sums over $j$ implicitly are understood only to be a sum over sites on the same molecule as site $i$, because the two sites involved in an intramolecular bond must belong to the same molecule.

The intermolecular association strength is per mole, and for sPC-SAFT the expressions for $\Delta^{\text {inter }}$ and $W_{n}$ in eqs 5 and 7 must therefore be corrected with Avogadro's number.

The starting point is the function $Q$ which is equal to the Helmholtz free energy at the stationary point with respect to the site fractions $X$, given by:

$$
\begin{aligned}
& Q / n=\sum_{k}^{\mathrm{NC}} x_{k}\left(\ln X 0_{k}+K_{k}+1-\sum_{i}^{\mathrm{NS}} X A_{i}\right) \\
& -\frac{1}{2 V_{m}} \sum_{i} \sum_{j} x_{i} x_{j} X A_{i} X A_{j} \Delta_{i j}^{\mathrm{inter}}-\frac{1}{2} \sum_{i} \sum_{j \neq i} x_{i} X A B_{i j} \Delta_{i j}^{\mathrm{intra}}
\end{aligned}
$$

with

$$
K_{k}=X 0_{k} \sum_{P\left(\Gamma_{k}\right)=\{\gamma, M \geq 2\}}(-1)^{M}(M-2) ! \prod_{\gamma_{k}} \frac{X_{\Gamma_{k}-\gamma_{k}}}{X 0_{k}}
$$

$X 0_{k}$ is the monomer fraction of component $k, \mathrm{NC}$ is the number of components, and $\mathrm{NS}_{k}$ is the number of sites on component $k$.

The $Q$ function has the property that all derivatives with respect to any bonding state, $X_{\alpha}$, are zero:

$$
\frac{\partial Q}{\partial X_{\alpha}}=0
$$

and in the stationary point:

$$
\begin{aligned}
Q_{\mathrm{sp}}= & \frac{A^{\mathrm{assoc}}}{R T}=\sum_{k}^{\mathrm{NC}} x_{k}\left(\ln X 0_{k}-\sum_{i}^{\mathrm{NS}}\left(X A_{i}-1\right)\right) \\
& -\frac{1}{2} \sum_{i} \sum_{j \neq i} x_{i} X A B_{i j} \Delta_{i j}^{\mathrm{intra}} \\
-1+ & X A_{i}\left[1+\frac{1}{V_{m}} \sum_{j} x_{j} X A_{j} \Delta_{i j}^{\mathrm{inter}}\right]+\sum_{j \neq i} X A B_{i j} \Delta_{i j}^{\mathrm{intra}}=0
\end{aligned}
$$

and

$$
\begin{aligned}
K_{k}= & -1+\sum_{i}^{\mathrm{NS}} X A_{i}-\frac{n}{V} \sum_{i}^{\mathrm{NS}} \sum_{j} x_{j} X A_{i} X A_{j} \Delta_{i j}^{\mathrm{inter}} \\
& -\frac{1}{2} \sum_{i}^{\mathrm{NS} S_{k}} \sum_{j \neq i} X A B_{i j} \Delta_{i j}^{\mathrm{intra}}
\end{aligned}
$$

The derivation of eqs $13-15$ is shown in the paper by Avlund et al. $^{5}$

The pressure is obtained from the volume derivative of the Helmholtz free energy:

$$
\frac{P^{\text {assoc }}}{R T}=-\frac{\partial Q_{\mathrm{pp}}}{\partial V}
$$

The derivative of $Q$ is calculated using the chain rule: ${ }^{36}$

$$
\frac{\partial Q_{\mathrm{sp}}}{\partial V}=\left(\frac{\partial Q}{\partial V}\right)_{X}+\sum_{k} \sum_{\alpha \subset \Gamma_{k}} \frac{\partial Q}{\partial X_{\alpha}} \frac{\partial X_{\alpha}}{\partial V}=\left(\frac{\partial Q}{\partial V}\right)_{X}
$$

and thus

$$
\begin{aligned}
& -\frac{P^{\text {assoc }}}{R T}=\frac{-n^{2}}{2 V}\left(-\frac{1}{V} \sum_{i} \sum_{j} x_{i} x_{j} X A_{i} X A_{j} \Delta_{i j}^{\text {inter }}\right. \\
& \left.\quad+\sum_{i} \sum_{j} x_{i} x_{j} X A_{i} X A_{j} \frac{\partial \Delta_{i j}^{\text {inter }}}{\partial V}\right)-\frac{n}{2} \sum_{i} \sum_{j} x_{i} X A B_{i j} \frac{\partial \Delta_{i j}^{\text {intra }}}{\partial V}
\end{aligned}
$$

$\Delta^{\text {inter }}$ and $\Delta^{\text {intra }}$ only depend on volume (and composition) through the radial distribution function $g$. The volume derivative of $\Delta^{\text {inter }}$ is:

$$
\frac{\partial \Delta^{\text {inter }}}{\partial V}=\frac{\Delta^{\text {inter }}}{g} \frac{\partial g}{\partial V}=\Delta^{\text {inter }} \frac{\partial \ln g}{\partial V}
$$

and similarly for $\Delta^{\text {intra }}$. Using this and eq 14 , we can simplify the expression for the pressure:

$$
\begin{aligned}
-\frac{P^{\text {assoc }}}{R T}= & \frac{n^{2}}{2 V^{2}} \sum_{i} \sum_{j} x_{i} x_{j} X A_{i} X A_{j} \Delta_{i j}^{\text {inter }} \\
& -\left(\frac{n^{2}}{2 V} \sum_{i} \sum_{j} x_{i} x_{j} X A_{i} X A_{j} \Delta_{i j}^{\text {inter }}+\frac{n}{2} \sum_{i} \sum_{j} x_{i} X A B_{i j} \Delta_{i j}^{\text {intra }}\right) \\
& \times \frac{\partial \ln g}{\partial V}=\frac{n^{2}}{2 V^{2}} \sum_{i} \sum_{j} x_{i} x_{j} X A_{i} X A_{j} \Delta_{i j} \\
& -\frac{n}{2} \sum_{i} x_{i}\left(1-X A_{i}\right) \frac{\partial \ln g}{\partial V}
\end{aligned}
$$

The chemical potential of component $l$ is obtained from the compositional derivative of the Helmholtz free energy:

$$
\frac{\mu_{l}^{\text {assoc }}}{R T}=\frac{\partial Q_{\mathrm{sp}}}{\partial n_{l}}=\left(\frac{\partial Q}{\partial n_{l}}\right)_{X}+\sum_{k} \sum_{\alpha \subset \Gamma_{k}} \frac{\partial Q}{\partial X_{\alpha}} \frac{\partial X_{\alpha}}{\partial n_{l}}=\left(\frac{\partial Q}{\partial n_{l}}\right)_{X}
$$

We have to distinguish between associating and nonassociating components. For nonassociating components, we find:

$$
\begin{aligned}
\frac{\mu_{l}^{\text {assoc }}}{R T} & =-\left(\frac{n^{2}}{2 V} \sum_{i} \sum_{j} x_{i} x_{j} X A_{i} X A_{j} \Delta_{i j}^{\text {inter }}+\frac{n}{2} \sum_{i} \sum_{j \neq i} x_{i} X A B_{i j} \Delta_{i j}^{\text {intra }}\right) \frac{\partial \ln g}{\partial n_{l}} \\
& =-\frac{n}{2} \sum_{i} x_{i}\left(1-X A_{i}\right) \frac{\partial \ln g}{\partial n_{l}}
\end{aligned}
$$

and for associating components we have:

$$
\begin{aligned}
\frac{\mu_{l}^{\text {assoc }}}{R T} & =\ln X 0_{l} \\
& +\left[1+K_{l}-\sum_{i}^{\mathrm{NS}} X A_{i}-\frac{n}{V} \sum_{i}^{\mathrm{NS} S_{k}} \sum_{j} x_{j} X A_{i} X A_{j} \Delta_{i j}^{\mathrm{inter}}-\frac{1}{2} \sum_{i}^{\mathrm{NS} S_{k}} \sum_{j \neq i} X A B_{i j} \Delta_{i j}^{\mathrm{intra}}\right] \\
& -\left(\frac{n^{2}}{2 V} \sum_{i} \sum_{j} x_{i} x_{j} X A_{i} X A_{j} \Delta_{i j}^{\mathrm{inter}}+\frac{n}{2} \sum_{i} \sum_{j \neq i} x_{i} X A B_{i j} \Delta_{i j}^{\mathrm{intra}}\right) \frac{\partial \ln g}{\partial n_{l}} \\
& =\ln X 0_{l}-\frac{n}{2} \sum_{i} x_{i}\left(1-X A_{i}\right) \frac{\partial \ln g}{\partial n_{l}}
\end{aligned}
$$

The terms in the square bracket sum to zero according to eq 15 , and $\ln X 0_{l}$ is the only difference between the derivative for associating and nonassociating components.

More derivatives are given in the Ph.D. thesis by Avlund. ${ }^{4}$ 


\section{AUTHOR INFORMATION}

\section{Corresponding Author}

*E-mail: gk@kt.dtu.dk.

\section{ACKNOWLEDGMENT}

We wish to thank the Technical University of Denmark for the Ph. D. scholarship to A.S.A., as well as Statoil and Gassco (Norway), BP (UK, USA), Mærsk Oil and Gas, and DONG Energy (Denmark) for supporting this work as part of the CHIGP project (Chemicals in Gas Processing).

\section{REFERENCES}

(1) Jackson, G.; Chapman, W.; Gubbins, K. Phase equilibria of associating fluids. Spherical molecules with multiple bonding sites. Mol. Phvs. 1988, 65, 1-31.

(2) Chapman, W. G.; Jackson, G.; Gubbins, K. E. Phase equilibria of associating fluids: chain molecules with multiple bonding sites. Mol. Phys. 1988, 65, 1057-79.

(3) Chapman, W. G.; Gubbins, K. E.; Jackson, G.; Radosz, M. SAFT: Equation-of-state solution model for associating fluids. Fluid Phase Equilib. 1989, 52, 31-38.

(4) Avlund, A. S. Extension of association models to complex chemicals. Ph.D. thesis, Technical University of Denmark, 2011.

(5) Avlund, A. S.; Kontogeorgis, G. M.; Chapman, W. G. Intramolecular association within the SAFT framework. Mol. Phys. 2011, 109, 1759-1769.

(6) Missopolinou, D.; Panayiotou, C. On intramolecular and intermolecular hydrogen bonding. Fluid Phase Equilib. 1999, 156, 51-56.

(7) Missopolinou, D.; Ioannou, K.; Prinos, I.; Panayiotou, C. Thermodynamics of alkoxyethanol+alkane mixtures. Z. Phvs. Chem. 2002, 216, 905-918.

(8) Missopolinou, D.; Tsivintzelis, I.; Panayiotou, C. Phase compositions and saturated densities for the binary system of carbon dioxide with 2-ethoxyethanol. Fluid Phase Equilib. 2005, 238, 204-209.

(9) Missopolinou, D.; Tsivintzelis, I.; Panayiotou, C. Excess enthalpies of binary mixtures of 2-ethoxyethanol with four hydrocarbons at 298.15, 308.15, and 318.15K. Fluid Phase Equilib. 2006, 245, 89-101.

(10) Garrido, N.; Folas, G.; Kontogeorgis, G. Modelling of phase equilibria of glycol ethers mixtures using an association model. Fluid Phase Equilib. 2008, 273, 11-20.

(11) Garcia-Lisbona, M.; Galindo, A.; Jackson, G.; Burgess, A. Predicting the high-pressure phase equilibria of binary aqueous solutions of 1-butanol, n-butoxyethanol and n-decylpentaoxyethylene ether (C10E5) using the SAFT-HS approach. Mol. Phys. 1998, 93, 57-71.

(12) Von Solms, N.; Michelsen, M. L.; Kontogeorgis, G. M. Computational and physical performance of a modified PC-SAFT equation of state for highly asymmetric and associating mixtures. Ind. Eng. Chem. Res. 2003, 42, 1098-1105.

(13) Brinkley, R. L.; Gupta, R. B. Intra- and intermolecular hydrogen bonding of 2-methoxyethanol and 2-butoxyethanol in n-hexane. Ind. Eng. Chem. Res. 1998, 37, 4823-4827.

(14) Sear, R.; Jackson, G. Thermodynamic perturbation theory for association into chains and rings. Phvs. Rev. E 1994, 50, 386-94.

(15) Ghonasgi, D.; Chapman, W. Competition between intermolecular and intramolecular association in flexible hard chain molecules. I. Chem. Phvs. 1995, 102, 2585-2592.

(16) Huang, S.; Radosz, M. Equation of state for small, large, polydisperse, and associating molecules. Ind. Eng. Chem. Res. 1990, 29, 2284-2294.

(17) Yarrison, M.; Chapman, W. A systematic study of methanol + $n$-alkane vapor-liquid and liquid-liquid equilibria using the CK-SAFT and PC-SAFT equations of state. Fluid Phase Eauilib. 2004, 226, 195-205.
(18) Avlund, A. S.; Eriksen, D. K.; Kontogeorgis, G. M.; Michelsen, M. L. Application of association models to mixtures containing alkanolamines. Fluid Phase Equilib. 2011, 306, 31-37.

(19) Rowley, R.; Wilding, W.; Oscarson, J.; Yang, Y.; Giles, N. DIPPR Data Compilation of Pure Chemical Properties: Design Institute for Physical Properties; AIChE: New York, NY, 2010.

(20) Gross, J.; Sadowski, G. Perturbed-chain SAFT: An equation of state based on a perturbation theory for chain molecules. Ind. Eng. Chem. Res. 2001, 40, 1244.

(21) Dolch, E.; Matovu, A.; Lichtenthaler, R. VLE and LLE in binary 2-methoxyethanol/alkane systems. Fluid Phase Equilib. 1986, 29, 257-264.

(22) Bijl, H.; Deloos, T. W.; Lichtenthaler, R. N. Liquid-liquid equilibria in 2-methoxyethanol + alkane systems. 1. Experimental results at pressures up to $400 \mathrm{MPa}$. Fluid Phase Equilib. 1985, 22, 321-342.

(23) Murti, P.; Van Winkle, M. Vapor-liquid equilibria and heat of mixing - normal-octane-ethylbenzene-cellosolve system. AICHE I. 1957, $3,517-522$.

(24) Rubio, M.; Gonzalez, J.; de la Fuente, I.; Cobos, J. Thermodynamic properties of $\mathrm{n}$-alkoxyethanols plus organic solvent mixtures. IX. Liquid-liquid equilibria of systems containing 2-methoxyethanol or 2-ethoxyethanol and selected n-alkanes. I. Chem. Eng. Data 1998, 43, 811-814.

(25) Komatsu, H.; Suzuki, I.; Ishikawa, T.; Mizukami, K. Kogyo Kagaku Zasshi 1969, 72, 1417.

(26) Suresh, S. J.; Elliott, J. Multiphase equilibrium analysis via a generalized equation of state for associating mixtures. Ind. Eng. Chem. Res. 1992, 31, 2783-2794.

(27) Grenner, A.; Kontogeorgis, G.; von Solms, N.; Michelsen, M. Modeling phase equilibria of alkanols with the simplified PC-SAFT equation of state and generalized pure compound parameters. Fluid Phase Equilib. 2007, 258, 83-94.

(28) Chandak, B.; Nageshwar, G.; Mene, P. Vapor-liquid-equilibria, excess enthalpy and volume and viscosity of normal-propanol-methyl cellosolve. Indian I. Technol. 1977, 15, 146-149.

(29) Grenner, A.; Schmelzer, J.; von Solms, N.; Kontogeorgis, G. M. Comparison of two association models (Elliott-Suresh-Donohue and Simplified PC-SAFT) for complex phase equilibria of hydrocarbonwater and amine-containing mixtures. Ind. Eng. Chem. Res. 2006, 45, $8170-8179$.

(30) Chiavone-Filhi, O.; Proust, P.; Rasmussen, P. Vapor-liquid equilibria for glycol ether + water systems. I. Chem. Eng. Data 1993, $38,128-131$.

(31) Boublik, T.; Kuchynka, K. Chem. Listy 1956, 50, 1181.

(32) Schneider, G.; Wilhelm, G. Z. Phvs. Chem. 1959, 20, 219.

(33) Aizpiri, A.; Monroy, F.; del Campo, C.; Rubio, R.; Peña, M. Range of simple scaling and critical amplitudes near a LCST - the 2-butoxyethanol plus water-system. Chem. Phys. 1992, 165, 31-39.

(34) Schneider, G. Drückeinflüss auf die Entmischung flüssiger Systeme, I Geschlossene Mischungslücken bis 5000 bar. Z. Phvs. Chem. (Muenchen. Ger.) 1963, 37, 333-352.

(35) Knudsen, K.; Stenby, E. H.; Andersen, J. G. Modeling the influence of pressure on the phase-behavior of systems containing water, oil and nonionic surfactants. Fluid Phase Equilib. 1994, 93, 55-74.

(36) Michelsen, M.; Hendriks, E. Physical properties from association models. Fluid Phase Equilib. 2001, 180, 165-174. 